\title{
The New Antiepileptic Drugs or the Traditional Antiepileptic Drugs? Update in Terms of Drug Side Effects
}

\author{
Ibrahim Bora ${ }^{\mathrm{a}}$, Aylin Bican Demir ${ }^{\mathrm{a}} \mathrm{b}$
}

\begin{abstract}
Epilepsy is defined as episodic cerebral dysfunction due to increased excitability of brain cells that caused by various reasons. Anti-epileptic drugs (AED) cannot stop the mechanisms that cause epilepsy, but they can decrease the frequency of seizures or completely stop the seizures without causing a general depression in central nervous system while they are using. In this case report, we aimed to discuss the therapeutic way that physician chose, depending on personal experiences in patient that developed adverse effects to both old generation (phenytoin, valproic acid) and new generation (Vaigabatrine, Lamotirgine, Levetiracetam, Pregabaline) AEDs. Even though it needs to be careful when treating a patient with epilepsy to maximize the therapeutic efficacy and to prevent complications, we intended to emphasise that there are unknown parts of AED mechanisms.
\end{abstract}

Keywords: Antiepileptic drugs; Epilepsy; Mechanism; Side effect; Toxicity

\section{Introduction}

Epilepsy is defined as episodic cerebral dysfunction due to increased excitability of brain cells that caused by various reasons [1]. Epilepsy is a common disease and one of important disorders that affect daily life and life quality. It's prevalence is $5-10$ per 1000 people [2, 3]. Anti-epileptic drugs (AED) cannot stop the mechanisms that cause epilepsy but they can decrease the frequency of seizures or completely stop the seizures without causing a general depression in

Manuscript accepted for publication December 14, 2012

${ }^{a}$ Uludag University Medical Faculty, Department of Neurology, Bursa, Turkey

${ }^{\mathrm{b}}$ Corresponding author: Aylin Bican Demir, Uludag University Medical School Neurology, Uludag Universitesi Tip Fakultesi, Noroloji ABD, 16059 /Gorukle, Bursa, Turkey. E-mail: aylinbd@uludag.edu.tr

doi: http://dx.doi.org/10.4021/jnr167e central nervous system while they are using. Mostly, AEDs increase the inhibition or decrease neuronal excitability. They generate membrane stability by reducing membrane excitability to resting membrane potential $[4,5]$.

Similar to all other long-term medications, there is a risk to develop adverse effects against AEDs. Severe hematological, gastrointestinal, endocrine, skin and aesthetical adverse effects may develop [6, 7]. Novel adverse effects due to long-term use of AEDs are being discussed in recent studies on case report basis. Therefore, a physician must be informed and alert for adverse effects during follow ups of patients AEDs using for a long time. We aimed to present a case report who developed adverse effects to various AEDs, including conventional and new generation drugs.

\section{Case Report}

Thirty-eight-year-old housewife, female patient have had her first seizure at the 7th month when she was hospitalized in neonatal care unit for meningitis, and she was being followed by Uludag University Medical Faculty Department of Neurology Epilepsy Clinic since. Physicians started her on phenobarbital therapy and she developed seizures like staring eyes, smacking lips, lasting for few seconds at age of 1.5. Phenobarbital therapy was continued and she developed seizures for $4-5$ times in a month as looking with feared eyes, screaming, focal initiated and secondary generalized contractions starting from right arm and leg and generalized to whole body at age of 2. Valproic acid and clonazepam drops added to therapy. She had no seizures till 12 years old and patients mentioned that she had delayed neuromotor development and was sent to a special education program. The frequency of secondary generalized seizures initiating from right leg and arm increased after age of 12 . Her valproic acid and rivotril therapy was stopped, and carbamezapine was added to her therapy in the beginning. Her seizures persisted, and vigabatrine was added to her therapy. Parents mentioned that her seizures went under control for 3 - 4 years with this treatment regimen. The patient was followed closely for vigabatrine use, and vigabatrine was stopped because of visual field disorder development. Frequency of seizures increased 
and Phenytoin was added to carbamezapine treatment. With this treatment regimen, she had $1-2$ seizures per year in 5 - 6 years period. During this period she developed gingival hypertrophy, but her parents did not want to change the therapy. Therefore phenytoin and carbamezapine doses were reduced. Topiramate $50 \mathrm{mg} /$ day was added to therapy, and patient developed focal motor seizures on right arm and right leg for 4 - 5 times a week. Therefore Valproic acid (1000 mg/day) was added to therapy. Because patient's focal motor seizures persisted with Valproic acid $100 \mathrm{mg} /$ day and topiramate $150 \mathrm{mg}$ /day therapy, Lamotrigine was added to her therapy with titration starting dose of $25 \mathrm{mg} / \mathrm{day}$. During titration process, patient's platelet value started to decrease from 92,000 to 84,000 . She was consulted with haematology department and diagnosed as idiopathic thrombocytopenia, and then Lamotrigine therapy was stopped. Levetiracetam $1000 \mathrm{mg} /$ day was added to therapy as third agent. During follow ups, patien's platelet values continued to decrease progressively(102,000 - 99,000). We decided to hospitalize the patient, and her platelet value was 46,000 at the time of hospitalization.

Diffuse cortical atrophy and bilateral hippocampal atrophy was observed in the control MRI (Fig. 1). A Spike wave activity was observed at left frontotemporal area in EEG (Fig. 2). A mini mental test was performed. The patient had $13 / 30$ score and reported as cognitive disorder at moderate level. Blood Valproic acid level was very high at $170 \mathrm{mg} /$ $\mathrm{dl}$ and blood ammonia level was normal. Valproic acid was suspected as responsible of symptoms, thus the dose was reduced and eventually stopped. Initially, platelet value increased to 72,000 , and then decreased to 48,000 afterwards. Levetiracetam was suspected as responsible of thrombocytopenia, and the dose was reduced and stopped eventually. She was started on pregabalin $150 \mathrm{mg} / \mathrm{day}$, and the dose was increased to $300 \mathrm{mg} /$ day. Platelet values increased to 192,000 , and persisted at this levels during follow ups. Patient's seizures were under control with topiramate $100 \mathrm{mg}$ /day and pregabalin $600 \mathrm{mg} /$ day therapy.

Our patient mentioned that she feeled good and was discharged from hospital. Patient's persisting symptoms with ongoing therapy were restless leg, and tension symptom with increased degrees during bedtime and decreasing with walking. The patient was internalized again with these symptoms and her laboratory tests resulted as $9.4 \mathrm{gr} / \mathrm{dl} \mathrm{Hbg}$, $10 \mathrm{Fe}$, normal Fe binding capacity and ferritin. Secondary reasons that can cause Fe deficiency was investigated, and an abdominal USG was performed and resulted as normal. Haematology department diagnosed our patient as Fe deficiency anemia, and her replacement therapy was planned. Polysomnography (PSG) and suggested immobilisation tests were performed (Fig. 3). Periodic legs movements disorders (PLMD) was resulted as 34 per hour in PSG test, and she was diagnosed as secondary restless leg syndrome due to Fe deficiency anemia. Pramipexole $0.25 \mathrm{mg} 2 \mathrm{X} 1$ and $\mathrm{Fe}$ replacement therapies were administered. Her restless leg syndrome was significantly reduced during hospitalization. She mentioned about a weight increase from $57 \mathrm{~kg}$ to $75 \mathrm{~kg}$ in her last treatment. Her topiramate dose was increased due to increase of weight and seizures with frequency of $3-4$ times in a month. Topiramate dose was increased to $200 \mathrm{mg} /$ day. The patient mentioned that her restless leg syndrome was significantly improved and she was satisfied with the therapy. Her last therapy was pramipexole $0.25 \mathrm{mg} 2$ times/ day, topiramate $200 \mathrm{mg} /$ day and pregabalin $600 \mathrm{mg} /$ day. Her seizures was very rare, as right sided, mild focal motor seizures. We decided to continue this treatment regimen and follow up closely.

\section{Discussion}

\section{Phenytoin}

Phenytoin was first synthesized in 1908, and put into use in 1937. It is one of the most common three agents for epilepsy treatment, with carbamezapine started to use in 1959 and valproate started to use in 1969. There are many novel agents that put on market in the last decade, like vigabatrine, lamotrigine, gabapentin, pregabalin, oxcarbazepine, topiramate, tiagabine, zonisamide and felbamate. Also, many of them are in ongoing clinical trials. The mechanism of action of these drugs (blocking of ion channels, increasing of GABAergic transmission, antagonizing of excitatory transmission) are in interaction with some other drug's mechanisms. Nevertheless the exact effects of these drugs at molecular basis are still unknown. Therefore, it is difficult to assess the clinical effectiveness of these drugs in different type of seizures. Lack of knowledge about activation mechanisms of these drugs may sometimes manifests itself as discovering novel adverse effects. Some of these drugs differs from previous agents with features like metabolic transformation and low interaction potential (vigabatrine and gabapentin). Some of these novel agents are metabolized intensively and plays a major role in drug-drug interaction $[8,9]$.

Antiepileptic treatment is a long-term and sometimes a lifelong treatment. Using these drugs at efficient doses may lead to some major adverse effects. Many chronic adverse effects are difficult to diagnose and sometimes cannot be diagnosed for years. This situation makes it difficult to approach a patient with possible toxicity.

Adverse effects developed in acute phase against AEDs' use are more commonly hypersensitivity reactions ralated and mostly dose depended. Adverse effects developed in chronic phase are various and difficult to diagnose. toxicity findings can be classified as local toxicity (gastrointestinal adverse effects), dose depended toxicity (sedation, elevation of liver function markers, cerebellar disorders) and idiosyncratic toxicity (anemia, thrombocytopenia, leucopenia) [10]. 
Phenitoin belongs to drug family, named as old drugs, like phenobarbital, benzodiazephines, and bromine, and has been used for nearly 75 years for treatment of focal seizures, primer and secondary generalized tonic clonic seizures worldwide [11]. Phenytoin's adverse effects are well known, and can be categorized as dose depended, idiosyncronic and cronic, depending on the knowledge collection with common use of phenytoin. Gingival hyperplasia is another chronic adverse effect of phenitoin use and could develop after 2 - 3 months of phenytoin use. significant gingival hyperplasia can be seen after 9 - 12 months of phenytoin use [12]. In a study of Arya et al, 120 pediatric patients that developed gingival hyperplasia due to phenytoin use were included and followed for 6 months. Sixty-two patients treated with folic acid and 58 patients with placebo. Gingival hyperplasia was found significantly reduced in patients treated with folic acid.

\section{Vigabatrine}

Vigabatrine (VGB), an efficient AED been used for a long time, is a selective irreversible inhibitor of GABA transaminase that is a breakdown product of aminobutyric acid (GABA) [13]. VGB is mostly well tolerated, but may cause adverse effects like unconsciousness, depression, increase of appetite, behavioral disorders, headache, urinary retention, constipation, double vision, visual field defects, skin rashes, ataxia, increase of weight, psychotic reactions. These adverse effects are mostly mild, temporary and dose depended. In a study of Moskowitz et al, 114 patients were treated with vigabatrine and developed visual field defects at various levels and went under electroretinography. The results revealed that 39 patients had unsignificant changes. But, in the remaining 76 patients, significant changes were observed at various levels. In some patients, these changes effected vision. Authors mentioned that visual field defect is irreversible and may result from toxic effect of VGB on retina [14]. Our patient did not want to stop using vigabatrine but we had to stop VGB due to its adverse effects on visual field.

\section{Lamotrigine}

Lamotrigine is a voltage depended $\mathrm{Na}$ channel blocking agent, and it has also a regulatory effect on $\mathrm{Ca}$ and potassium channels [15]. Some of well known adverse effects of Lamotrigine are as follow: nausea, vomitting, solomnance, drowsiness, ataxia, diplopia, headache, blurred vision, and tremor in high doses. An important adverse effect is systemic skin rashes and it can progress to Stevensen-Johnson syndrome (must be carefull with pediatric patients) and toxic epidermal necrosis. We found a case report during literature research of a 45 -year-old patient with partial seizures who developed thrombocytopenia and leucopenia during Lamotrigine titration. The authors mentioned that he also had haematological adverse effect with Phenobarbital use. Patient's thrombocye and leucocyte levels reached to normal following lamotrigine dose reduction and cease of lamotrigine. They stated that this phenmenon may arise from drugdrug interaction or an unknown adverse effect of lamotrigine [15]. We had to stop lamotrigine, because our patient developed thoeombocyte level recuction during lamotrigine titration while on Valproic acid and Topiramate therapy.

\section{Valproic acid}

It was demonstrated that valproic acid affects as antiepileptic drug via affecting $\mathrm{Na}^{+}$channels, $\mathrm{Ca}^{++}$flow and increasing GABAergic activity. It was also demonstrated that valproic acid inhibits GABA-transaminase enzyme that brakes down GABA, and increases the activity of glutamic acid decarboxylase that plays role in GABA synthesis [16]. Valproic acid may cause gastrointestinal adverse effects like nausea, anorexia, dyspepsia, diarrhea, constipation, and dose depended heamatological adverse effects like thrombocytopenia, trombocyte function detoriation and leucopenia, as well as various other adverse effects like increase of weight, hair loss, skin rashes, tremor and sedation [17]. Elevated plasma levels of AEDs may rarely cause increase in frequency of seizures. AED ensephalopatia might been developed if other central nervous system toxicity findings are exists. This increase in frequency of seizures associated with Valproic acid (VPA) use may be seen as increase in the frequency of existing seizures or non epileptic negative myoclonus type seizures. Hepatotoxicity, toxic ensephalopaty (hyperamoniaemia or normal serum values) may be involved with increase in frequency seizures associated with VPA [18]. In a study about VPA and toxicity, it has been stated that haematologi-

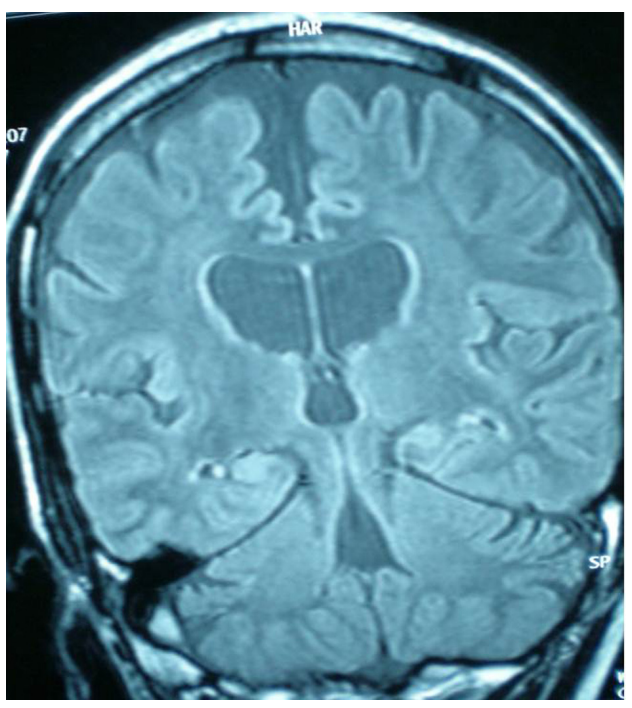

Figure 1. Diffuse cortical atrophy and bilateral hippocampal atrophy was observed in the control MRI. 


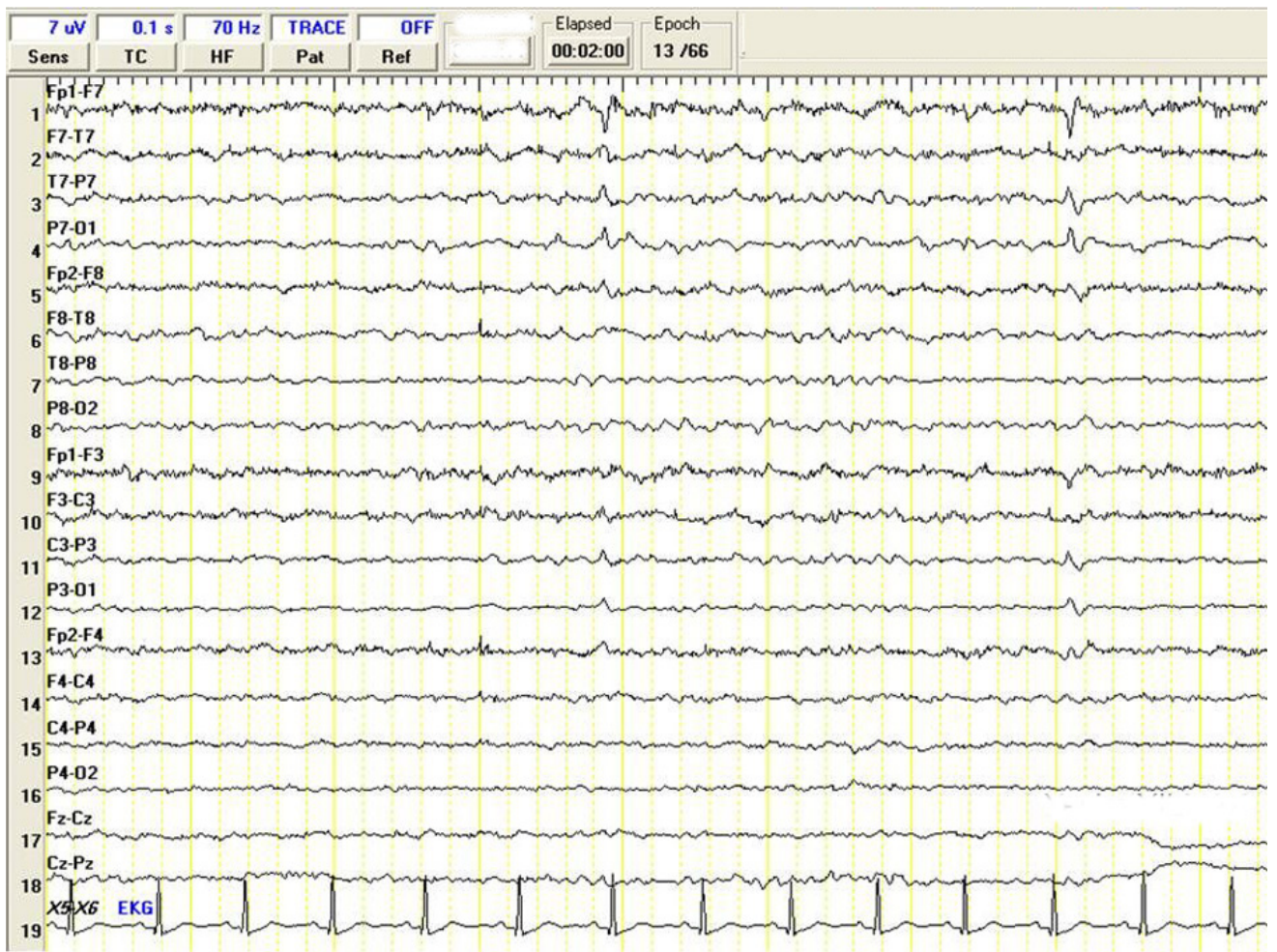

Figure 2. A Spike wave activity was observed at left frontotemporal area in EEG.

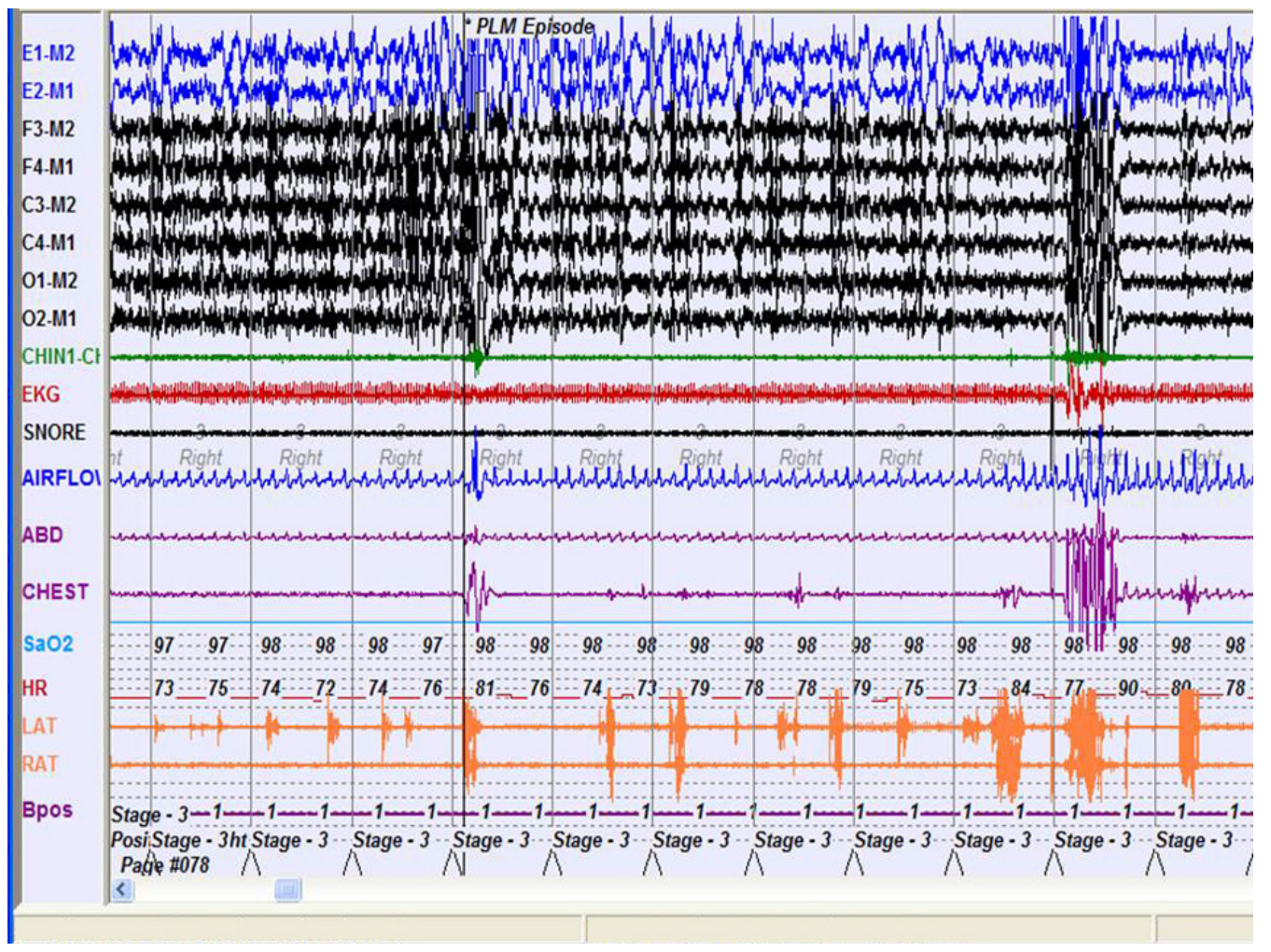

Figure 3. Polysomnography (PSG) and suggested immobilisation tests were performed. 
cal toxicity associated with VPA is common and can be seen at various levels. Haematological toxicity may be temporary or permanent. The authors also mentioned that physicians must be careful with blood level of VPA $>100$ microgram/ $\mathrm{mL}$ [17]. In our patient VPA blood level was 170 microgram/ $\mathrm{mL}$ and blood ammoniac level was normal. During follow ups, our patient had idiopathic thrombocytopenia and we thought VAP might been associated with this phenomenon. VPA dose was decreased and stopped eventually. Thrombocyte level started to increase. But after a while, it started to decrease again and this time another AED LEV was considered as responsible from this phenomenon. Therefore, LEV dose was decreased and stopped eventually. Patient's platelet level reached to normal levels during follow ups.

\section{Levetiracetam}

Levetiracetam (LEV) is believed to have effect on high voltage, $\mathrm{N}$ type calcium channels and regulatory effect on potassium flow [11]. Last studies about LEV demonstrated that the agent increases the GABAergic activity via regulating protein $2 \mathrm{a}$, which is a synaptic protein involved in vesicle exocytosis mechanism. Fatigue, somnolence, headache, nausea, amnesia and ataxia are most common adverse effects of LEV. Other adverse effects related to LEV use are as follow: diarrhea, loss of appetite, depression, vertigo, dyspepsia, emotional fluctuations, insomnia, aggression, tremor, temper, loss of balance, skin rashes, diplopia, leucopenia, neutropenia, pancytopenia, thrombocytopenia, behavioral disorders, anger, anxiety, convulsion, hallucination, irritability, psychotic disorders. In a retrospective study of Sahaya et al, it has been stated that twenty-nine of 758 Levetirasetam using epilepsy patients developed thrombosytopenia due to unknown mechanism and this phenomenon is reversible [19]. As mentioned above, our patient's platelet level reached to normal following the cease of LEV use.

\section{Pregabalin}

Pregabalin is a structural analogue of GABA (delta-aminobutyric acid) but does not affect via GABA receptors. Pregabalin does not activated by potassium channels, sodium channels and glutamate receptors. Pregabalin affects as gabapentin like via binding alfa 2-delta subunit of $\mathrm{P} / \mathrm{Q}$ type voltage sensitive calcium channels (VSCC) in presynaptic neurons. Pregabalin's analgesic, anxiolytic and anticonvulsant effects were demonstrated in various animal studies [20].

In a open-labeled, multiple dose application study of Brodie et al, $200 \mathrm{mg} / 3$ times in a day (a total of $600 \mathrm{mg}$ ) for 7 days pregabalin was added as second agent to patients using valproate (VPA), phenytoin (PHT), lamotrigine (LTG) or carbamezapine (CBZ) as monotherapy. And no drug-drug interactions was observed with other AEDs [21]. Nevertheless, in another study, it has been stated that pregabalin level decreases approximately $20-30 \%$ with use of enzyme inducing AED (such as carbamezapine). The most common adverse effects are somnolence and drawsiness, and followed by headache, vertigo, dry mouth and increase of weight [22]. We could not find any case report about restless leg and $\mathrm{Fe}$ deficiency as a cause of restless leg associated with pregabalin.

In the case that we were following up closely, the patient responded to pregabalin therapy, but restless leg syndrome also developed with pregabalin use. We searched literature about mechanisms that can cause restless leg syndrome via dopaminergic mechanisms other than binding alfa 2-delta subunit of $\mathrm{P} / \mathrm{Q}$ type voltage sensitive calcium channels (VSCC) in presynaptic neurons. Nevertheless we could not find any related mechanism. We hypothesized that pregabalin may affect neurons at molecular or neurotransmitter level other than binding alfa 2-delta subunit of $\mathrm{P} / \mathrm{Q}$ type voltage sensitive calcium channels (VSCC), since our patient dramatically responded to dopamine antagonists and developed symptoms shorty after pregabalin use.

In this case report we aimed to discuss the therapeutic way that physician chose depending on personal experiences, in patient that developed adverse effects to both old generation (phenytoin, valproic acid) and new generation (Vaigabatrine, Lamotirgine, Levetiracetam, Pregabaline) AEDs. Even though it needs to be careful while treating a patient with epilepsy to maximize the therapeutic efficacy and to prevent complications, we intended to emphasise that there are unknown parts of AEDs mechanisms.

\section{References}

1. Engel J, Jr. A proposed diagnostic scheme for people with epileptic seizures and with epilepsy: report of the ILAE Task Force on Classification and Terminology. Epilepsia. 2001;42(6):796-803.

2. Sander J.W. E-epilepsy-Library of articles-The incidence and prevalence of epilepsy.The National Society for Epilepsy 2003.

3. Calisir N, Bora I, Irgil E, Boz M. Prevalence of epilepsy in Bursa city center, an urban area of Turkey. Epilepsia. 2006;47(10):1691-1699.

4. Depondt C. The potential of pharmacogenetics in the treatment of epilepsy. Eur J Paediatr Neurol. 2006;10(2):57-65.

5. Katzung BG. Basic \& Clinical Pharmacology USA; Mc Graw Hill; 2001; 395-419.

6. McNamara JO. Emerging insights into the genesis of epilepsy. Nature. 1999;399(6738 Suppl):A15-22.

7. Schmidt D, Elger C, Holmes GL. Pharmacological overtreatment in epilepsy: mechanisms and management. Epilepsy Res. 2002;52(1):3-14.

8. Brodie MJ, Kwan P. Newer drugs for focal epilepsy in 
adults. BMJ. 2012;344:e345.

9. Fattore C, Perucca E. Novel medications for epilepsy. Drugs. 2011;71(16):2151-2178.

10. Privitera M. Current challenges in the management of epilepsy. Am J Manag Care. 2011;17 (Suppl 7):S195203.

11. Armijo JA, Shushtarian M, Valdizan EM, Cuadrado A, de las Cuevas I, Adin J. Ion channels and epilepsy. Curr Pharm Des. 2005;11(15):1975-2003.

12. Arya R, Gulati S, Kabra M, Sahu JK, Kalra V. Folic acid supplementation prevents phenytoin-induced gingival overgrowth in children. Neurology. 2011;76(15):13381343.

13. Griffin WC, 3rd, Nguyen SA, Deleon CP, Middaugh LD. Effects of vigabatrin, an irreversible GABA transaminase inhibitor, on ethanol reinforcement and ethanol discriminative stimuli in mice. Behav Pharmacol. 2012;23(2):178-190.

14. Moskowitz A, Hansen RM, Eklund SE, Fulton AB. Electroretinographic (ERG) responses in pediatric patients using vigabatrin. Doc Ophthalmol. 2012;124(3):197-209.

15. Siniscalchi A, Gallelli L, Calabro G, Tolotta GA, De Sarro G. Phenobarbital/Lamotrigine coadministrationinduced blood dyscrasia in a patient with epilepsy. Ann Pharmacother. 2010;44(12):2031-2034.

16. Macdonald RL, Kelly KM. Mechanisms of action of currently prescribed and newly developed antiepileptic drugs. Epilepsia. 1994;35(Suppl 4):S41-50.

17. Acharya S, Bussel JB. Hematologic toxicity of sodium valproate. J Pediatr Hematol Oncol. 2000;22(1):62-65.

18. Gerstner T, Buesing D, Longin E, Bendl C, Wenzel D, Scheid B, Goetze G, et al. Valproic acid induced encephalopathy--19 new cases in Germany from 1994 to 2003--a side effect associated to VPA-therapy not only in young children. Seizure. 2006;15(6):443-448.

19. Sahaya K, Goyal MK, Sarwal A, Singh NN. Levetiracetam-induced thrombocytopenia among inpatients: a retrospective study. Epilepsia. 2010;51(12):2492-2495.

20. Vartanian MG, Radulovic LL, Kinsora JJ, Serpa KA, Vergnes M, Bertram E, Taylor CP. Activity profile of pregabalin in rodent models of epilepsy and ataxia. Epilepsy Res. 2006;68(3):189-205.

21. Brodie MJ, Wilson EA, Wesche DL, Alvey CW, Randinitis EJ, Posvar EL, Hounslow NJ, et al. Pregabalin drug interaction studies: lack of effect on the pharmacokinetics of carbamazepine, phenytoin, lamotrigine, and valproate in patients with partial epilepsy. Epilepsia. 2005;46(9):1407-1413.

22. Randinitis EJ, Posvar EL, Alvey CW, Sedman AJ, Cook JA, Bockbrader HN. Pharmacokinetics of pregabalin in subjects with various degrees of renal function. J Clin Pharmacol. 2003;43(3):277-283. 